\title{
To the question for formation of a block-modular structure of the control system for unmanned aerial vehicles
}

\author{
I.V. Kovalev ${ }^{1,2}$, V.V. Losev ${ }^{2, *}$, M.V. Saramud ${ }^{1,2}$, A.O. Kalinin', A.S. Lifar ${ }^{2}$ \\ ${ }^{1}$ Siberian Federal University, Krasnoyarsk, Russia \\ ${ }^{2}$ Reshetnev university, Krasnoyarsk, Russia \\ *E-mail: basilos@mail.ru
}

\begin{abstract}
The article discusses an approach to the formation of the basic structure of the control system for unmanned aerial vehicles, based on existing system solutions, typical specifications of the developer's boards. The characteristics of an unmanned aerial vehicle of both air and space class are given. General requirements for hardware and peripheral devices of unmanned aerial vehicles have been formed in accordance with their basic operating modes. Based on the obtained and generalized information about the developer's boards, the block-modular structure of the control system for unmanned aerial vehicles is presented. It includes all the main elements of the system and can be expanded by connecting additional boards. The advantage of this structure for debugging purposes is the presence of an FPGA on the development board.
\end{abstract}

Keywords: unmanned aerial vehicle, control system, peripherals 


\title{
К вопросу формирования блочно-модульной структуры системы управления беспилотных летательных объектов
}

\author{
И.В. Ковалев ${ }^{1,2}$, В.В. Лосев ${ }^{2, *}$ М.В. Сарамуд ${ }^{1,2}$, А.О. Калинин ${ }^{2}$, \\ А.С. Лифарь ${ }^{2}$ \\ ${ }^{1}$ Сибирский федеральный университет, Красноярск, Россия \\ ${ }^{2}$ СибГУ им. М.Ф. Решетнева, Красноярск, Россия \\ *E-mail: basilos@mail.ru
}

\begin{abstract}
Аннотация: Встатье рассматривается подход к формированию базовой структуры системы управления беспилотными летательными объектами, основанный на существующих системных решениях, типовых спецификациях плат разработчика. Дана характеристика беспилотному летательному объекту как воздушного, так и космического класса. Сформированы общие требования к аппаратного обеспечению и периферийным устройствам беспилотных летательных объектов согласно их базовым режимам работы. На основании полученной и обобщенной информации о платах разработчика представлена блочно-модульная структура системы управления беспилотных летательных объектов. Она включает в себя все основные элементы системы и может расширяться за счет подключения дополнительных плат. Преимуществом данной структуры для целей отладки может служить наличие ПЛИС на плате разработчика.
\end{abstract}

Ключевые слова: беспилотный летательный объект, система управления, периферийные устройства

\section{1. Введение}

Беспилотный летательный объект (далее - БЛО) - это объект, способный перемещаться или корректировать свое позиционирование в пространстве на основании некоторого набора входных данных (изображение с камер, сканирующих лидаров, показания датчиков, заранее заданный маршрут с привязкой к местности) и способный либо выполнять манипуляции с окружающими объектами, либо собирать/отправлять данные [1]. У подобных объектов опционально имеется возможность перехвата управления вручную, по каналу связи, однако это не всегда удобно, либо вовсе не реализуемо (например, работа в среде, в которой невозможно прохождение радиосигналов и обеспечение иных способов реализации канала связи). Определяющая характеристика - способность полностью автономной работы, без участия оператора или поступления иных команд извне.

Условно БЛО можно разделить на следующие классы: 
- Воздушные. К этому классу относятся как классические крылатые беспилотники и ракетная техника, так и набирающие популярность мультироторные системы. Отсутствие пилота позволяет кардинально снизить вес и уменьшить габариты летательного аппарата, соответственно снижаются и затраты на производство и сами полеты, в большинстве случаев отпадает необходимость в топливных двигателях, достаточно электромоторов, работающих от аккумуляторов. Небольшие габариты и отсутствие топливных двигателей - источников большого количества тепла существенно усложняют обнаружение подобных летательных средств. Основным недостатком беспилотных летательных аппаратов (БПЛА) считается уязвимость радиоканала связи для удаленного управления, автономный режим решает эту проблему, поскольку даже полная потеря канала не выводит аппарат из строя, он продолжает выполнять задачу. Отсутствие радиоканала также является существенным преимуществом в плане сложности обнаружения объекта. На данный момент воздушные БЛО находят все большее применение в коммерческом использовании, в сельском и лесном хозяйстве, в геодезии и картографии, практикуется доставка потребительских товаров с помощью автономных беспилотных мультироторных систем,

- Космические. К данному классу относятся как решения для постоянного нахождения в открытом космосе (спутники на околоземной орбите), так и аппараты, запускаемые на другие космические тела, предназначенные для передвижения по ним и активных действий - сбор и анализ образцов поверхности, атмосферы, фото, видеосъемка с дальнейшей отправкой результатов на землю. Реализация радиоканала связи для управления подобными объектами крайне затруднительна, поскольку на космических расстояниях начинает иметь значение скорость радиосигнала, внося существенные задержки как в сторону передачи управляющих команд, так и в сторону отправки видео или иного сигнала с управляемого объекта. Например, вы пытаетесь управлять объектом в трех световых минутах от земли, который постоянно транслирует картинку с камеры. В данный момент вы видите картинку не в реальном времени, а с задержкой не менее трех минут; отправляете управляющие команды, которые также дойдут до объекта только спустя 3 минуты, он их выполнит, а видеосигнал процесса выполнения дойдет до вас только спустя 6 минут после ваших команд на земле. Этот фактор существенно усложняет удаленное управление, даже при наличии стабильного канала связи, к тому-же расходует лишнюю энергию на аппарате для поддержания постоянной двусторонней связи на таком расстоянии. 
Поэтому космические аппараты разрабатываются автономными с возможностью ручного управления во внеплановых ситуациях [2]. На сегодняшний день дальние космические полеты осуществимы только беспилотными аппаратами, поскольку отправить человека на расстояние нескольких световых лет и вернуть его назад практически неосуществимо на данном этапе развития технологий. Наличие человека для подобных миссий не требуется, а его отсутствие позволяет сделать космический аппарат существенно легче, компактнее и проще, что очень важно для космических запусков. Благодаря миниатюризации электронных компонентов стали возможны такие проекты, как кубсат (англ. CubeSat) и покетсат (англ. PocketSat), что дает шанс на реальный запуск в космос студенческих малых спутников.

Таким образом, в основу формирования необходимого функционала плат разработчика положим БЛО воздушного и космического класса, как наиболее требовательные к надежности и габаритам.

\section{2. Общие требования к аппаратному обеспечению беспилотных летательных}

\section{объектов}

В процессе изучения функциональных узлов БЛО наиболее эффективным подходом является использования отладочных плат и плат разработчика. Плата разработчика средство для прототипирования и апробации автоматических систем управления и робототехники, ориентированное на широкий круг пользователей. Аппаратная часть представляет собой одноплатную структуру с минимально необходимым набором “обвязки” для нормальной работы микроконтроллера (программатор, стабилизатор питания, кварцевый резонатор, цепочки сброса и т. п.). Также имеется возможность подключения плат расширения для дополнения ее функционала. С точки зрения разработчика, программная часть состоит из программной оболочки (IDE) для написания программ, их компиляции и программирования аппаратуры. Плата разработчика может использоваться как для создания автономных объектов, так и подключаться к другим системам через стандартные проводные и беспроводные интерфейсы.

Среди существющих плат разработчика, были проанализированы платы, отвечающие таким требованиям, как: малое энергопотребление, открытая спецификация, подробная документация, поддержка распространенных языков программирования, официальная продажа на территории Российской Федерации, наличие возможности подключения внешних модулей расширения, поддержка операционной системы реального времени. 


\section{3. Анализ типовых узлов платы разработчика}

Проведенная спецификация и структурный анализ плат разработчика (Приложение 1, Таблица 1), позволяет выделить основные узлы систем (плат), которые формируют базовую (типовую) аппаратную платформу БЛО (класс воздушные) и являются ее неотъемлемой частью с возможностью последующей отладки [3,4]. Узлы имеют обобщенную спецификацию по назначению, представленную на рисунке 1 [5].
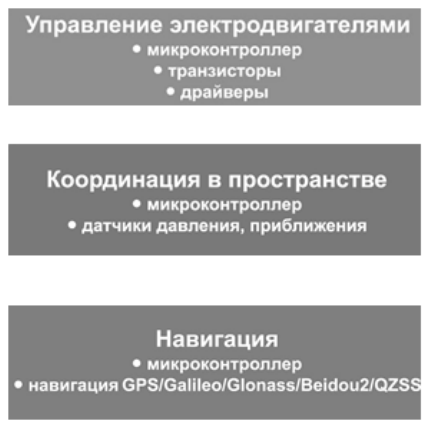

Стабилизация полета - датчики (акселеромтетры, гироскопь, магнитометры)

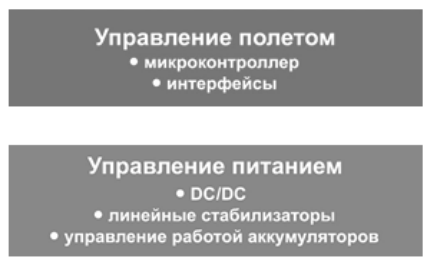

Защита от столкновений - датчики приближения

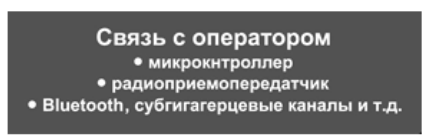

Рисунок 1. Основные узлы БЛО (класс воздушные) по назначению.

\section{1. Микропроцессор}

Основным узлом типового решения является сам микропроцессор, включающий в себя основную схемотехническую часть. В качестве примера типового решения можно привести такие процессоры как ARM Cortex-A53, ATmega16U2, ARM Cortex ${ }^{\mathrm{TM}}$ A8, Atom T5700, Altera Nios II Gen2, MKE15Z256VLH7.

\section{2. Память}

Вторым по важности компонентом является блок внешней памяти, позволяющий обрабатывать и хранить большое количество информации. Микроконтроллеры (МК) для своего функционирования требуют наличия нескольких видов памяти. FMC (Flexible Memory Controller), что обеспечивает параллельный доступ к памяти (NOR, PSRAM) за счет увеличения количество использованных для интерфейса пинов.

Для операций, не требующих высокой скорости доступа к памяти, можно использовать последовательные интерфейсы:

- Шина I2C, как правило, 100 или 400 кбит/с внешним 8-контактный EEPROM (энергонезависимая память); 
- SPI-шина, обычно 10 Мбит с использованием 4 проводов для подключения к EEPROM или Flash (NOR или NAND);

- SDIO шина и QSPI 1, 2 или 4 (или более) линии передачи данных, чтобы увеличить пропускную способность данных с внешней Flash.

SDIO, I2C, SPI - все обрабатывается встроенным программным обеспечением в режиме чтения и записи.

\section{3. Порты ввода/вывода}

Для прототипа БЛО третьим по важности элементом является наличие портов ввода/вывода для аналоговых и цифровых сигналов, наличие аналого-цифрового преобразователя (АЦП) и цифро-аналогового преобразователя (ЦАП) для них, наличие широтно-импульсной модуляция (ШИМ) и т.д.

\section{4. Часы реального времени}

Для бесперебойного функционирования также необходимо наличие часов реального времени. Данный модуль необходим для протоколирования данных со штампом времени и восстановления после сбоя в аварийном режиме. К каждой записи показаний на SD-карту или на удалённый сервер возможно добавление штампа времени. GPS-приёмники могут ускорять процесс включения, сравнивая время в собственных часах реального времени со временем последнего получения сигнала от спутника (если предыдущее включение было несколько часов назад, то ранее найденные спутники всё ещё могут быть использованы и определение координат начнется быстрее). Как правило, данные модули обеспечиваются автономным питанием.

\section{5. Таймеры}

Существует несколько видов таймеров, применяемых в микроконтроллерах. К примеру, 8-ми разрядный таймер общего назначения содержит предделитель, который ведет обратный отсчет от системных часов. Выходной сигнал предделителя поступает в счетчик, который отсчитывает от него сохраненное в нем значение. Когда счетчик опустошается, устанавливается флаг, и может выполняться прерывание. Существуют системы захвата входных данных и системы сравнения выходных данных, содержащие до 16 таймеров [6]. В моделях высокого класса существует блок процессора таймера, у которого время является основным операндом. Одним из значимых видов таймеров является сторожевой таймер (WDT), который вызывает сброс микроконтроллера. Это позволяет восстановить нормальную работу микроконтроллера вследствие 
кратковременных сбоев, вызванных перегрузкой, влиянием электромагнитного импульса или некорректной работой управляющей программы.

\section{6. Последовательный ввод / вывод}

На сегодняшний день относительно низкоскоростные асинхронные последовательные интерфейсы (SCI) используются для терминальных, модемных и сетевых интерфейсов. Высокоскоростные синхронные последовательные каналы (SPI) используются, в том числе для межкомпьютерных соединений, аппаратной периферийной связи и других типов устройств, где требуется высокоскоростная и безопасная связь.

Обычно системы SCI могут обмениваться данными на любой из популярных асинхронных последовательных скоростей передачи данных. Эти системы имеют встроенные генераторы скорости передачи данных, регистры ввода и вывода с двойной буферизацией, а также все средства обнаружения ошибок, собранные на универсальной микросхеме асинхронного приемопередатчика (UART). Эти устройства ввода-вывода могут опрашиваться или управляться прерываниями компьютерной частью микроконтроллера.

SPI предназначен для связи на высоких скоростях с другими микроконтроллерами или, возможно, с аппаратными устройствами с синхронным последовательным интерфейсом. Поскольку для синхронных систем требуются системные часы, каждый SPI микроконтроллера может действовать как ведущий или ведомый. Связь с микроконтроллером и SPI может быть опрашиваемой (синхронной) или через контроллер прерываний (асинхронной) [6].

\section{3. Периферийные устройства БЛО}

\section{1. Инерциальные датчики на основе МЭМС}

Сегодня датчики, основанные на технологии МЭМС (микроэлектромеханические системы), многочисленны по типам, что приводит к постоянному росту их применения. Эта технология используется при реализации критически важных инерциальных датчиков. Концепция вибрационного гироскопа Кориолиса является основой этой технологии при реализации гироскопов, также акселерометры всех типов подпадают под эту технологию. Текущий рывок в недорогой интегрированной инерциальной навигации в аэрокосмической отрасли, в первую очередь, связан с наличием миниатюрных приемников GPS и инерциальных систем на основе МЭМС [7, 8].

\section{2. Электродвигатели}


В мультироторных БПЛА силовая установка основана на системе прямого привода. Каждая стойка напрямую подключается к собственному источнику подъемной силы, в основном это бесщеточные электродвигатели. Большинство современных бесщеточных электродвигателей идентифицируются по ширине и высоте корпуса двигателя. Еще одна характеристика бесщеточных электродвигателей - номинальное напряжение в киловольтах. Это позволяет определить число оборотов двигателя на каждый вольт подаваемого в него электричества. Данное измерение выполняется при нулевой нагрузке на двигатель, поэтому в действительности, частота вращения будет варьироваться в зависимости от трения и нагрузки. Аккумулятор с более высоким напряжением заставит двигатель вращаться быстрее, но номинальное напряжение в киловольтах останется прежним.

\section{3. Канал телеметрии}

Для обеспечения двусторонней связи с БЛО необходима организация канала телеметрии с управляемым объектом путем формирования специальной двусторонней радиосвязи. Канал телеметрии берет серию измерений с управляемого объекта и отправляет их обратно на землю, где они могут быть отображены в программном обеспечении наземного управления. В качестве параметров канала телеметрии могут выступать: путевые точки, автономные полеты, конфигурации БЛО, а также широта, долгота, высота, положение БЛО, текущее напряжение батареи, курс БЛО, скорость, продолжительность полета и многое другое [9].

На сегодняшний день аэрофото-, видеосъемка является первоочередной востребованной задачей применения БПЛА. К примеру, наиболее часто используемые датчики на сельскохозяйственных БПЛА - это красный, зеленый и синий в полосе пропускания изображения в ближнем и инфракрасном диапазонах.

Конструктивно, датчики должны быть простыми в установке и отсоединении. Они должны быть достаточно миниатюрными, чтобы вместить полезную нагрузку и занять место на небольшом дроне. Современные тенденции заключаются в использовании небольших и быстрых дронов для получения аэрофотоснимков и сбора цифровых данных. Низколетящие и парящие БПЛА предпочтительнее, если требуются снимки крупным планом с более подробной детализацией объектов или местности [10].

\section{4. Позиционирование и навигация}

Отличительной особенностью между дроном и модельным самолетом является способность первого работать с использованием GPS. Добавление этой технологии спутниковой навигации обеспечивает уровень контроля, который раньше был просто 
Modern Innovations, Systems and Technologies, 2021, 1(3)

невозможен. Этот дополнительный контроль позволил конструкторам дронов разрабатывать режимы полета для конкретных типов полетов.

Режим «Лойтер» (Loiter) - позволяет удерживать дрон в определенном месте (широта, долгота и высота). При этом находясь в удержании положения дрон сам исправит свое месторасположение, если оно будет изменено какими-то внешними возмущениями.

Режим «Возврат домой» (RTH). Этот режим позволяет летательному аппарату определять домашнее местоположение, обычно исходное место взлета, и возвращаться туда при определенных условиях безотказной работы. Потеря радиосвязи с БПЛА - один из распространенных факторов, вызывающих возврат домой.

Режим «Навигация по путевым точкам». Это режим автономной навигации по запрограммированным путевым точкам. Программное обеспечение наземного управления загружает список инструкций по навигации в полетный контроллер, который выполняет их шаг за шагом, как полную миссию. Этот метод особенно полезен в индустрии картографирования, где необходимо снова и снова летать по определенным схемам полета.

Режим «Следуй за мной». Этот режим позволяет пользователю отправлять на дрон поток данных о местоположении пользователя в реальном времени с помощью мобильного приложения. Затем дрон использует данные о местоположении с вашего мобильного телефона, чтобы следовать за вами с заданной высотой и расстоянием [9].

\section{4. Блочно-модульная структуры системы управления БЛО}

На основании полученной и обобщенной информации о платах разработчика была составлена блочно-модульная структура, представленная на рисунке 2. Она включает в себя все основные элементы системы и может расширяться за счет подключения дополнительных плат. Дополнительным преимуществом для целей отладки может служить наличие ПЛИС на плате разработчика, но этот узел не является критически необходимым [11]. 


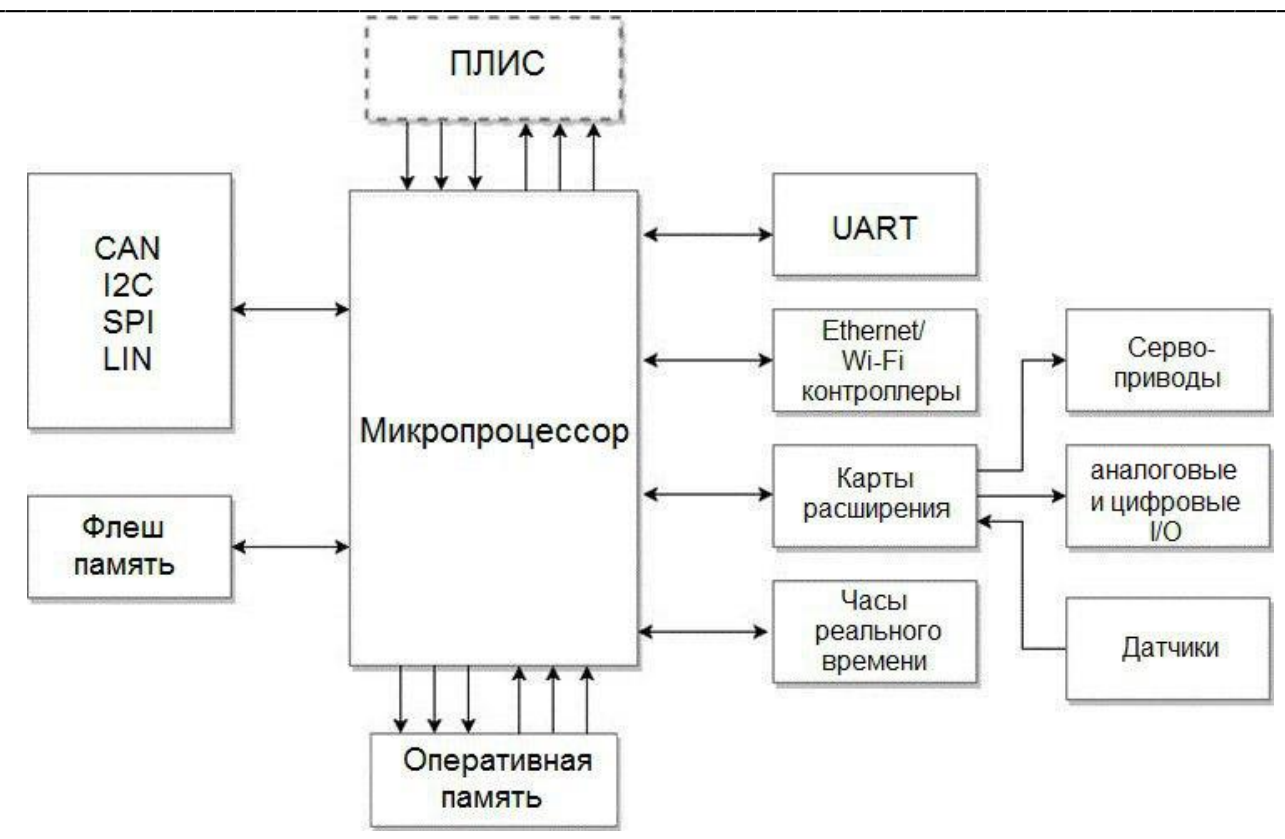

Рисунок 2. Блочно-модульная структура аппаратной платформы БЛО (класс воздушные).

\section{5. Заключение}

Большое число микропроцессоров, обладая разной архитектурой и периферией, подходят по всем сформированным требованиям и могут проявлять свою эффективность в различных ситуациях использования БЛО, что приводит к необходимости на этапе формирования требований к интегрированной среде разработки (IDE) включить в нее поддержку кроссплатформенности [12], т.е. поддержку большого числа микроконтроллеров, драйверов электродвигателей, датчиков и других элементов аппаратной части БЛО.

\section{Список литературы}

[1] Ковалев, И.В. К вопросу реализации мультиверсионной среды исполнения бортового программного обеспечения автономных беспилотных объектов средствами операционной системы реального времени / И.В. Ковалев, В.В. Лосев, М.В. Сарамуд, Д.И. Ковалев, М.О. Петросян // Вестник СибГАУ. - 2017. - № 1(18). - С. 58-61.

[2] Карцан, И. Построение наземных пунктов управления космическими аппаратами с использованием оптимизационно-имитационной модели. Современные инновации, системы и технологии / И. Карцан // Modern Innovations, Systems and Technologies. - 2021. - № 1(2). - C. 64-71. https://doi.org/10.47813/2782-2818-2021-12-64-71. 
[3] Аленченков, Г. С. Структурный синтез механизмов стартовых устройств беспилотных летательных аппаратов малой массы // Г.С. Аленченков, А.Э. Пушкарев. Интеллектуальные системы в производстве. - 2012. - № 1. - С. 5-11.

[4] Догерти, Мартин Дж. Дроны. Первый иллюстрированный путеводитель по БПЛА / Мартин Дж. Догерти. - М.: Издательство ЭКСМО, 2017. - 224 с.

[5] Функции электроники в составе мультикоптеров. Материал получен с сайта АО «КОМПЭЛ»-compel.ru (Дата обращения: 19.09.2021).

[6] Sickle, Ted Van. Programming Microcontrollers in C. Second edition / Ted Van Sickle // Newnes Publishing House, 2001. - P. 472.

[7] Bose, A., Fundamentals of navigation and initial sensors / A. Bose, K.H. Bhat, T. Kurian // PHI Learning. - 2014. - P. 322.

[8] Bose, A., S. Puri, Banerjee. Modern Inertial Sensors and Systems / A. Bose, S. Puri, Banerjee // PHI Learning. - 2008. - P. 416.

[9] Kilby, T. Make: Getting Started with Drones / T. Kilby, B. Kilby // Maker Media. - 2015. - P. 187.

[10] Krishna, K.R. Agricultural Drones. A Peaceful Pursuit / K.R. Krishna // Apple Academic Press. - 2018. - P. 381.

[11] Моисеев, В.С. Беспилотные авиационные комплексы. I. Структура и организация функционирования // В.С. Моисеев и др. Известия высших учебных заведений. Авиационная техника. - 2006. - №. 2. - С. 3-7.

[12] Ковалев, И., Сарамуд М., Лосев, В., Колташев, А. Метод и инструментарий верификации кроссплатформенного бортового программного обеспечения / И. Ковалев, М. Сарамуд, В. Лосев, А. Колташев // Современные инновации, системы и технологии - Modern Innovations, Systems and Technologies. - 2021. - № 1(2). - 22-33. https://doi.org/10.47813/2782-2818-2021-1-2-22-33.

\section{Приложение}

Таблица 1. Сравнительная таблица основных характеристик плат разработчика.

\begin{tabular}{|c|c|c|c|c|c|c|c|c|c|}
\hline 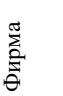 & Название & $\begin{array}{c}\text { Порты } \\
\text { ввода/выв } \\
\text { ода }\end{array}$ & ЦАП/АЦП & Интерфейсы & Встроенные модули & $\begin{array}{c}\text { Встроенны } \\
\text { е датчики }\end{array}$ & Память & $\begin{array}{c}\text { Архитектур } \\
\text { а ядра }\end{array}$ & IDE \\
\hline 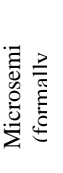 & $\begin{array}{c}\text { Smart } \\
\text { Fusion2 } \\
\text { Starter Kit }\end{array}$ & $\begin{array}{l}14 \text { - analog } \\
18 \text { - digital }\end{array}$ & $\begin{array}{l}\text { Configurable 8- } \\
\text { to 20-bit delta- } \\
\text { sigma ADC, Up } \\
\text { to two } 12 \text {-bit } \\
\text { SAR ADCs, } 4 \text { x } \\
\text { 8-bit DACs }\end{array}$ & $\begin{array}{c}\text { JTAG, Ethernet } \\
\text { 10/100, USB } \\
\text { OTG, SPI, I2C, } \\
\text { UART }\end{array}$ & $\begin{array}{c}\text { Ethernet } 10 / 100 \text {, USB } \\
\text { UART, USR OTG, } \\
\text { JTAG }\end{array}$ & - & $\begin{array}{l}\text { 64MB } \\
\text { LPDDR, } \\
\text { 16MB SPI } \\
\text { flash }\end{array}$ & 32 & - \\
\hline
\end{tabular}




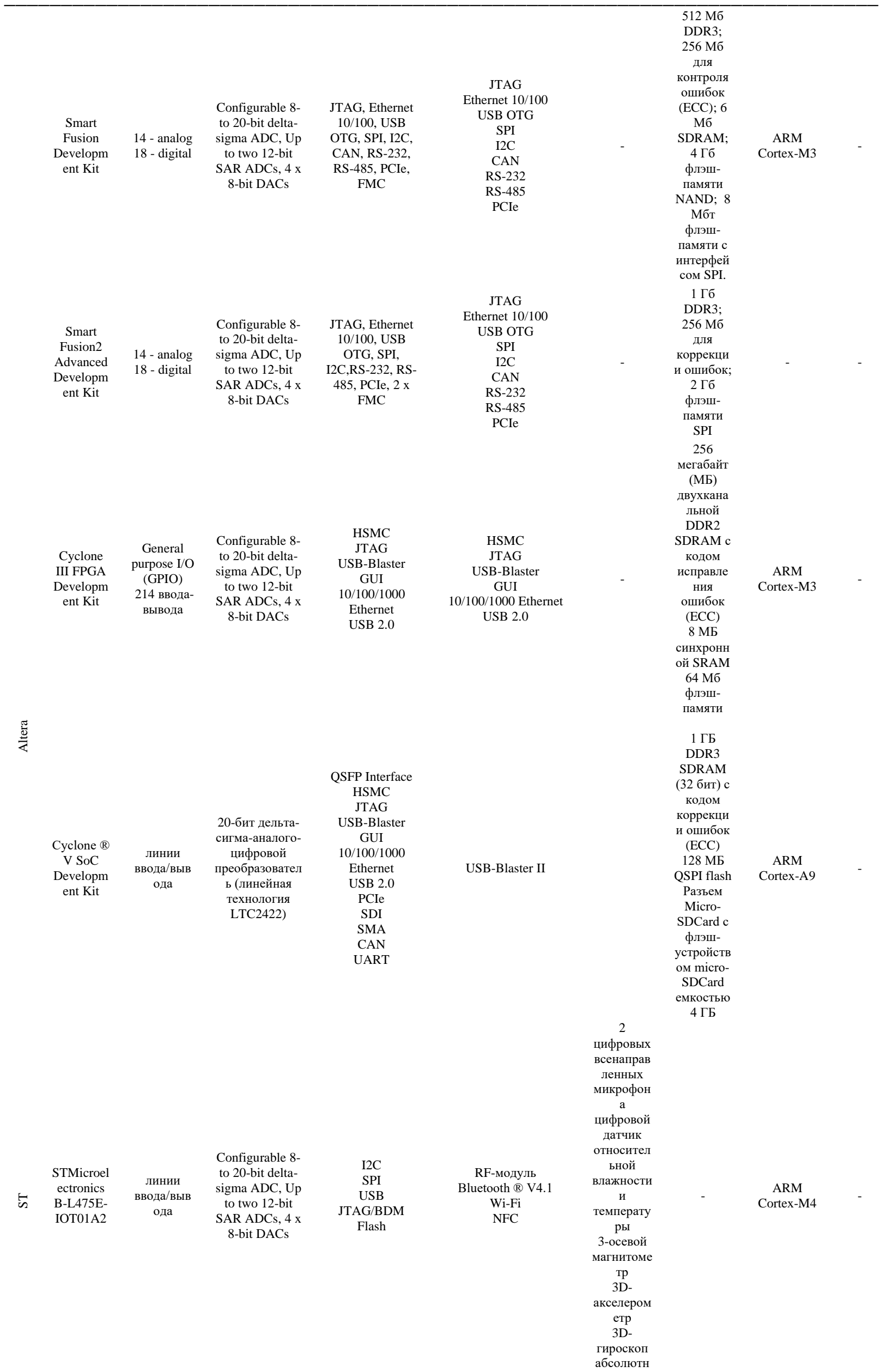




\begin{tabular}{|c|c|c|c|c|c|c|c|c|c|}
\hline & & & & & & $\begin{array}{c}\text { ый } \\
\text { цифровой } \\
\text { выходной } \\
\text { барометр } \\
\text { Датчик } \\
\text { времени } \\
\text { обнаружен } \\
\text { ия и } \\
\text { обнаружен } \\
\text { ия жестов }\end{array}$ & & & \\
\hline & $\begin{array}{l}\text { STMicroel } \\
\text { ectronics } \\
\text { P- } \\
\text { NUCLEO- } \\
\text { LRWAN1 }\end{array}$ & $\begin{array}{l}14 \text { - analog } \\
18 \text { - digital }\end{array}$ & $\begin{array}{l}\text { Configurable 8- } \\
\text { to 20-bit delta- } \\
\text { sigma ADC, Up } \\
\text { to two 12-bit } \\
\text { SAR ADCs, } 4 \text { x } \\
\text { 8-bit DACs }\end{array}$ & $\begin{array}{c}\text { I2C } \\
\text { SPI } \\
\text { USB } \\
\text { JTAG/BDM } \\
\text { UART }\end{array}$ & $\begin{array}{l}\text { ISM трансивер } \\
\text { LoRa трансивер }\end{array}$ & $\begin{array}{c}\text { температу } \\
\text { рный } \\
\text { датчик }\end{array}$ & - & Cortex-M0+ & - \\
\hline & $\begin{array}{l}\text { STM3210 } \\
\text { C-EVAL }\end{array}$ & $\begin{array}{l}14 \text { - analog } \\
18 \text { - digital }\end{array}$ & $\begin{array}{l}\text { вуковой ЦАП } \\
\text { I2S }\end{array}$ & $\begin{array}{c}\text { USB-OTG, } \\
\text { Ethernet, } \\
\text { CAN2.0A/B, I2S, } \\
\text { USART, SPI } \\
\text { RS-232 } \\
\text { irDA } \\
\text { JTAG }\end{array}$ & $\begin{array}{c}3.2 \text { "240х320 TFT } \\
\text { цветной ЖК-дисплей с } \\
\text { сенсорным экраном } \\
\text { Джойстик с } \\
\text { четырехпозиционным } \\
\text { управлением и } \\
\text { селектором } \\
\text { Кнопки сброса, } \\
\text { пробуждения, } \\
\text { вмешательства и } \\
\text { пользователя } \\
\text { IrDA- } \\
\text { приемопередатчик }\end{array}$ & - & $\begin{array}{c}\text { 64KB } \\
\text { SRAM } \\
\text { and } \\
\text { 256KB } \\
\text { Flash } \\
2 \text { ГБ } \\
\text { MicroSD } \\
\text { Card тм } \\
\text { или } \\
\text { больше }\end{array}$ & Cortex-M3 & - \\
\hline$\stackrel{\Xi}{\Xi}$ & $\begin{array}{l}\text { Atmel } \\
\text { ATEVK11 } \\
\text { 05AU }\end{array}$ & $\begin{array}{l}14 \text { - analog } \\
18 \text { - digital }\end{array}$ & $\begin{array}{l}\text { Configurable 8- } \\
\text { to 20-bit delta- } \\
\text { sigma ADC, Up } \\
\text { to two 12-bit } \\
\text { SAR ADCs, } 4 \text { x } \\
\text { 8-bit DACs }\end{array}$ & $\begin{array}{c}\text { I2C } \\
\text { SPI } \\
\text { TWI } \\
\text { USB } \\
\text { Ethernet } \\
\text { JTAG/BDM }\end{array}$ & $\begin{array}{c}\text { аудиоинтерфейс } \\
\text { сенсорный интерфейс }\end{array}$ & - & - & AVR32 UC & - \\
\hline & $\begin{array}{l}\text { Arty Artix- } \\
7 \text { FPGA }\end{array}$ & $\begin{array}{l}4 \text { Pmod } \\
\text { connectors, } \\
\text { Arduino/ } \\
\text { chip KIT } \\
\text { шилд } \\
\text { разъем; }\end{array}$ & $\begin{array}{l}\text { АЦП на } \\
\text { кристалле } \\
\text { (XADC), }\end{array}$ & $\begin{array}{l}\text { 10/ } 100 \mathrm{Mbps} \\
\text { Ethernet, } \\
\text { USB-UART } \\
\text { SPI } \\
\text { I2C } \\
\text { JTAG } \\
\text { PCIe }\end{array}$ & $\begin{array}{c}\text { 10/ } 100 \text { Mbps Ethernet, } \\
\text { USB-UART } \\
\text { SPI } \\
\text { I2C } \\
\text { JTAG } \\
\text { PCIe }\end{array}$ & & $\begin{array}{c}256 \mathrm{MB} \\
\text { DDR3L c } \\
16-\text { bit } \\
\text { шиной @ } \\
667 \mathrm{MHz}, \\
16 \mathrm{MB} \\
\text { Quad-SPI } \\
\text { Flash }\end{array}$ & & $\begin{array}{c}\text { Vivad } \\
\text { o } \\
\text { Desig } \\
\text { n } \\
\text { Suite }\end{array}$ \\
\hline$\stackrel{n}{E}$ & $\begin{array}{l}\text { Spartan-3E } \\
\text { Starter } \\
\text { Board }\end{array}$ & $\begin{array}{c}100- \\
\text { контактны } \\
\text { й разъем } \\
\text { Hirose FX2 } \\
\text { Три 6- } \\
\text { контактны } \\
\text { х порта } \\
\text { Pmod } \\
\text { DB15HD } \\
\text { VGA }\end{array}$ & видео ЦАП & $\begin{array}{l}\text { JTAG, Ethernet } \\
\text { 10/100, USB, } \\
\text { SPI, I2C,RS-232, } \\
\text { RS-485, PCIe, } \\
\text { VGA, PS/2, SMA }\end{array}$ & $\begin{array}{c}\text { SMSC LAN83C185 } \\
\text { Еthernet PHY } \\
\text { Клавиатура PS / } 2 \\
\text { RJ-45 Ethernet } \\
\text { 16-контактный разъем } \\
\text { для дополнительных } \\
\text { ЖК-модулей } \\
\text { Разъем SMA для } \\
\text { высокоскоростного } \\
\text { входного тактового } \\
\text { сигнала } \\
\text { Два разъема DB9 RS- } \\
232\end{array}$ & & $\begin{array}{c}64 \text { МБ } \\
\text { Micron }{ }^{\circledR} \\
\text { DDR } \\
\text { SDRAM } \\
\text { 16MB } \\
\text { Numonyx } \\
\text { StrataFlas } \\
\text { h TM } \\
\text { 2MB ST } \\
\text { Microelect } \\
\text { ronics }\end{array}$ & $\begin{array}{c}\text { Xilinx } \\
\text { Spartan-3E }\end{array}$ & \\
\hline$\ddot{s}$ & $\begin{array}{l}\text { CY8CKIT- } \\
001 \\
\text { PSoC } ® \\
\text { Developm } \\
\text { ent Kit }\end{array}$ & $\begin{array}{l}\mathrm{I} / \mathrm{O}(\mathrm{GPIO}) \\
\text { expansion } \\
\text { slots }\end{array}$ & $\begin{array}{l}\text { 12-bit Delta- } \\
\text { Sigma ADC, } 8- \\
\text { bit DAC }\end{array}$ & $\begin{array}{l}\text { USB 2.0, LCD, } \\
\text { JTAG, I2C, } \\
\text { SMBus, PMBus, } \\
\text { P15-DB9 Serial } \\
\text { Communication } \\
\text { Port, J9-Full- } \\
\text { Speed USB Port, } \\
\text { RS-232 }\end{array}$ & $\begin{array}{l}\text { P15-DB9 Serial } \\
\text { Communication Port, } \\
\text { J9-Full-Speed USB } \\
\text { Port, RS-232 }\end{array}$ & CapSense & $\begin{array}{c}\text { RAM } \\
64 \mathrm{~Kb}, \\
\text { Flash } \\
256 \mathrm{~KB}+ \\
32 \mathrm{~KB} \\
\text { Flash for } \\
\text { ECC, } \\
\text { EEPROM } \\
2 \mathrm{~KB}\end{array}$ & $\begin{array}{c}\text { M8C / 8051 / } \\
\text { Cortex-M3 }\end{array}$ & $\begin{array}{l}\text { PSoC } \\
\text { Creat } \\
\text { or } \\
\text { IDE }\end{array}$ \\
\hline 芯 & $\begin{array}{l}\text { CY8CKIT- } \\
050 \\
\text { PSoC® } \\
\text { 5LP } \\
\text { Developm } \\
\text { ent Kit }\end{array}$ & $\begin{array}{l}\mathrm{I} / \mathrm{O}(\mathrm{GPIO}) \\
\text { expansion } \\
\text { slots }\end{array}$ & $\begin{array}{l}\text { Configurable 8- } \\
\text { to 20-bit delta- } \\
\text { sigma ADC, Up } \\
\text { to two 12-bit } \\
\text { SAR ADCs, } 4 \text { x } \\
\text { 8-bit DACs }\end{array}$ & $\begin{array}{c}\text { RS-232 } \\
\text { I2C } \\
\text { SPI } \\
\text { USB } \\
\text { LCD } \\
\text { JTAG/BDM }\end{array}$ & $\begin{array}{l}\text { P15-DB9 Serial } \\
\text { Communication Port, } \\
\text { J9-Full-Speed USB } \\
\text { Port, RS-232 }\end{array}$ & CapSense & $\begin{array}{c}\text { RAM } \\
64 \mathrm{~Kb}, \\
\text { Flash } \\
256 \mathrm{~KB}+ \\
32 \mathrm{~KB} \\
\text { Flash for } \\
\text { ECC, } \\
\text { EEPROM } \\
2 \mathrm{~KB}\end{array}$ & $\begin{array}{c}\text { ARM } \\
\text { Cortex-M3 }\end{array}$ & $\begin{array}{l}\text { PSoC } \\
\text { Creat } \\
\text { or } \\
\text { IDE }\end{array}$ \\
\hline
\end{tabular}




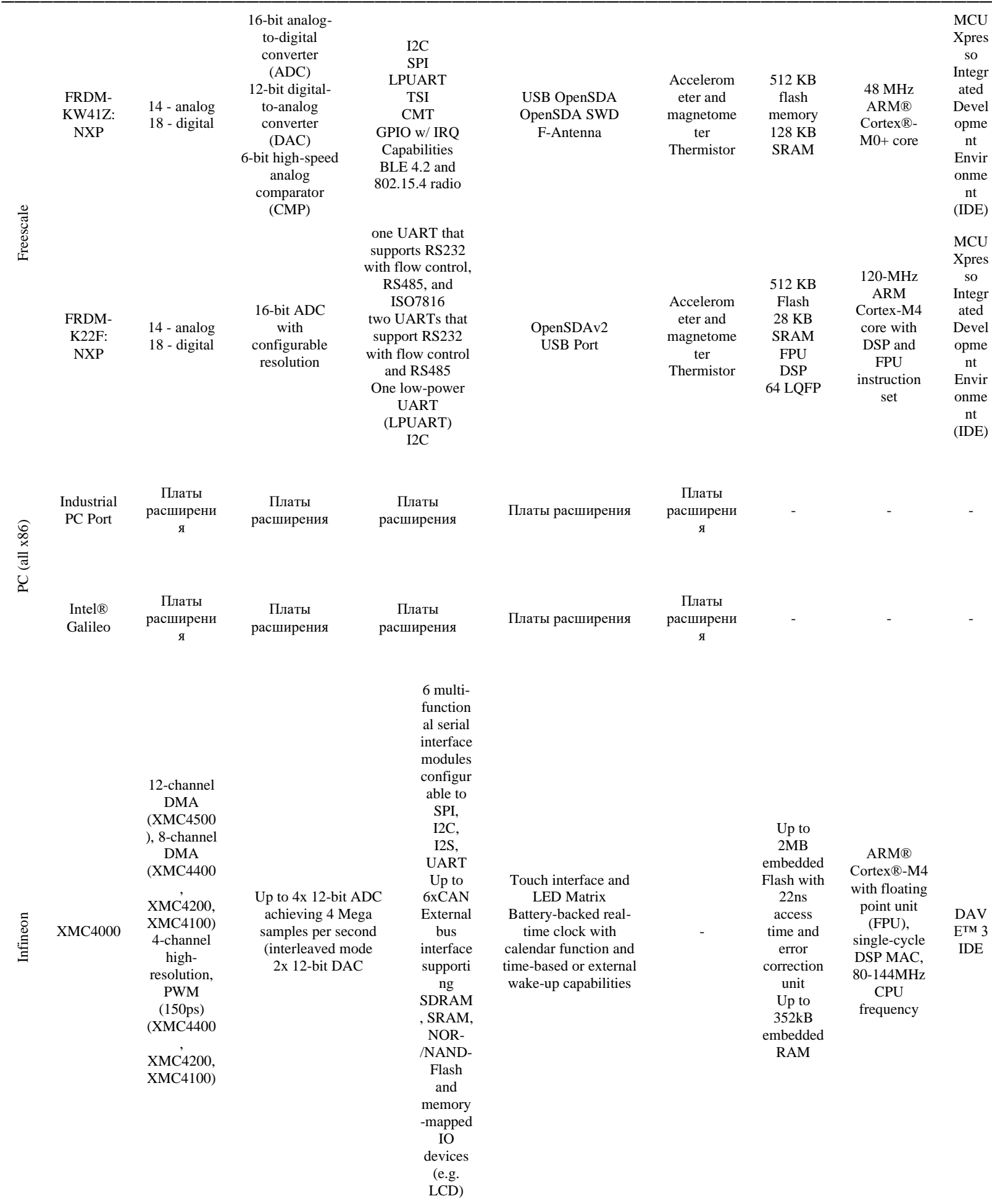




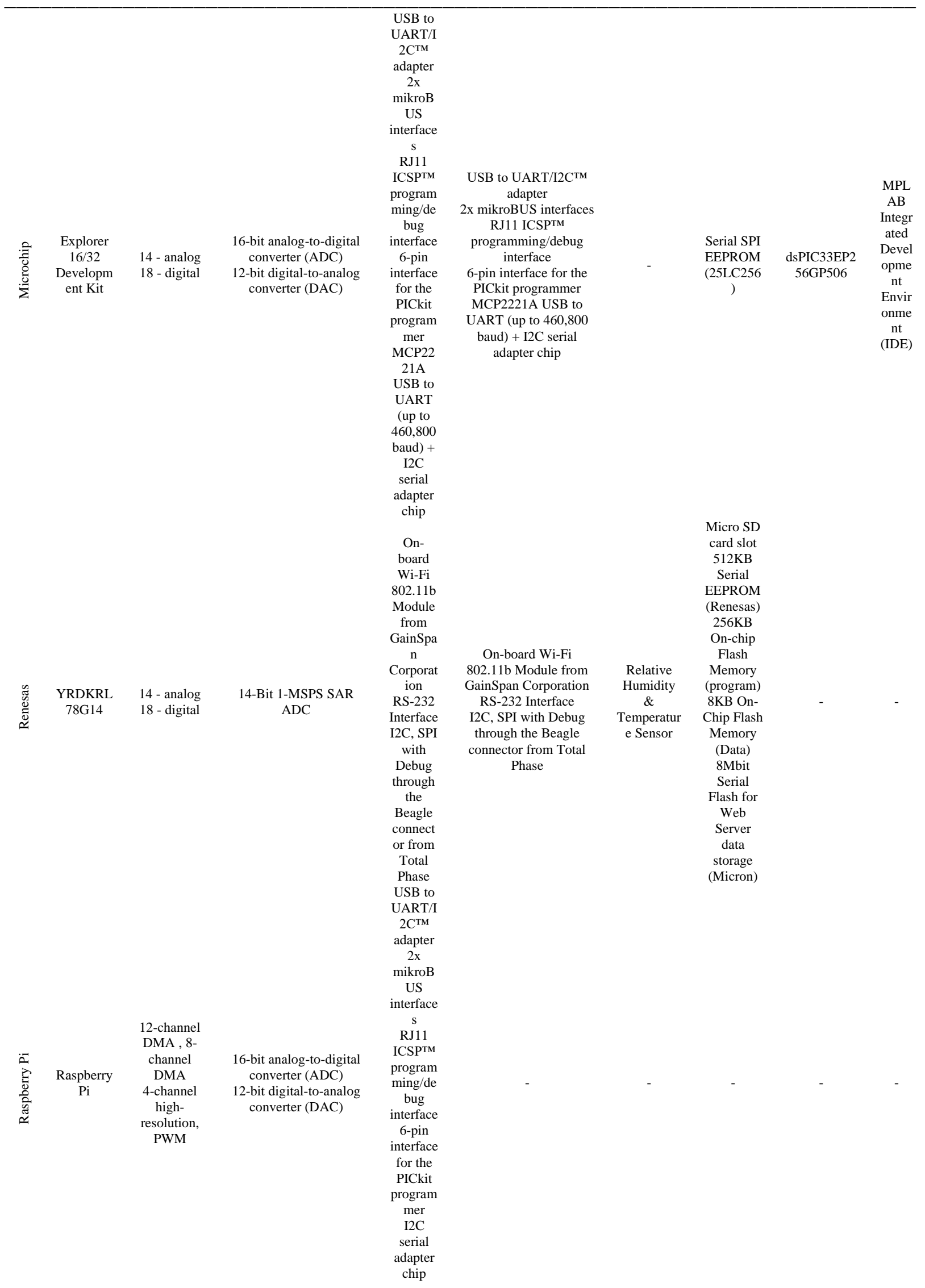




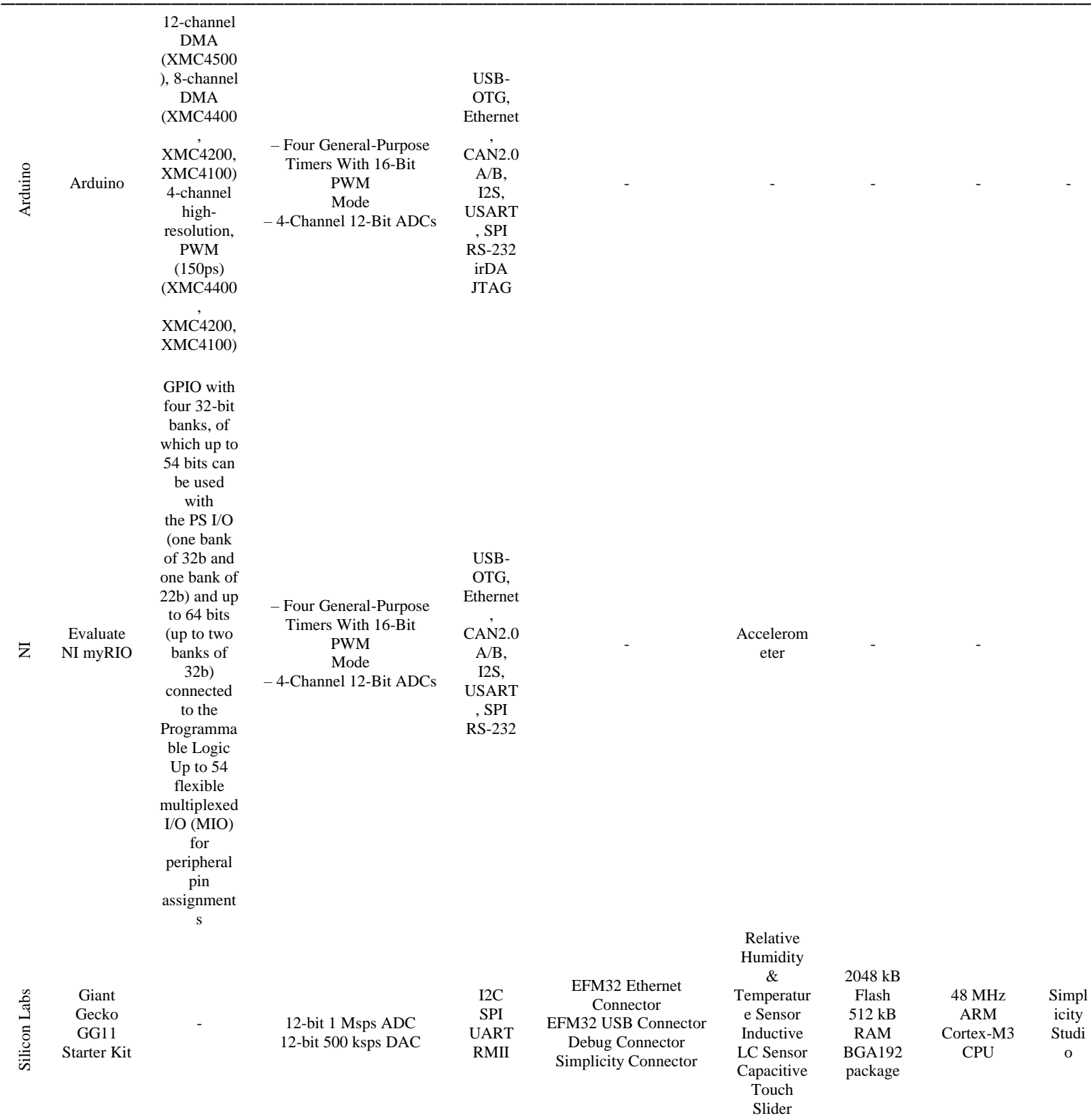




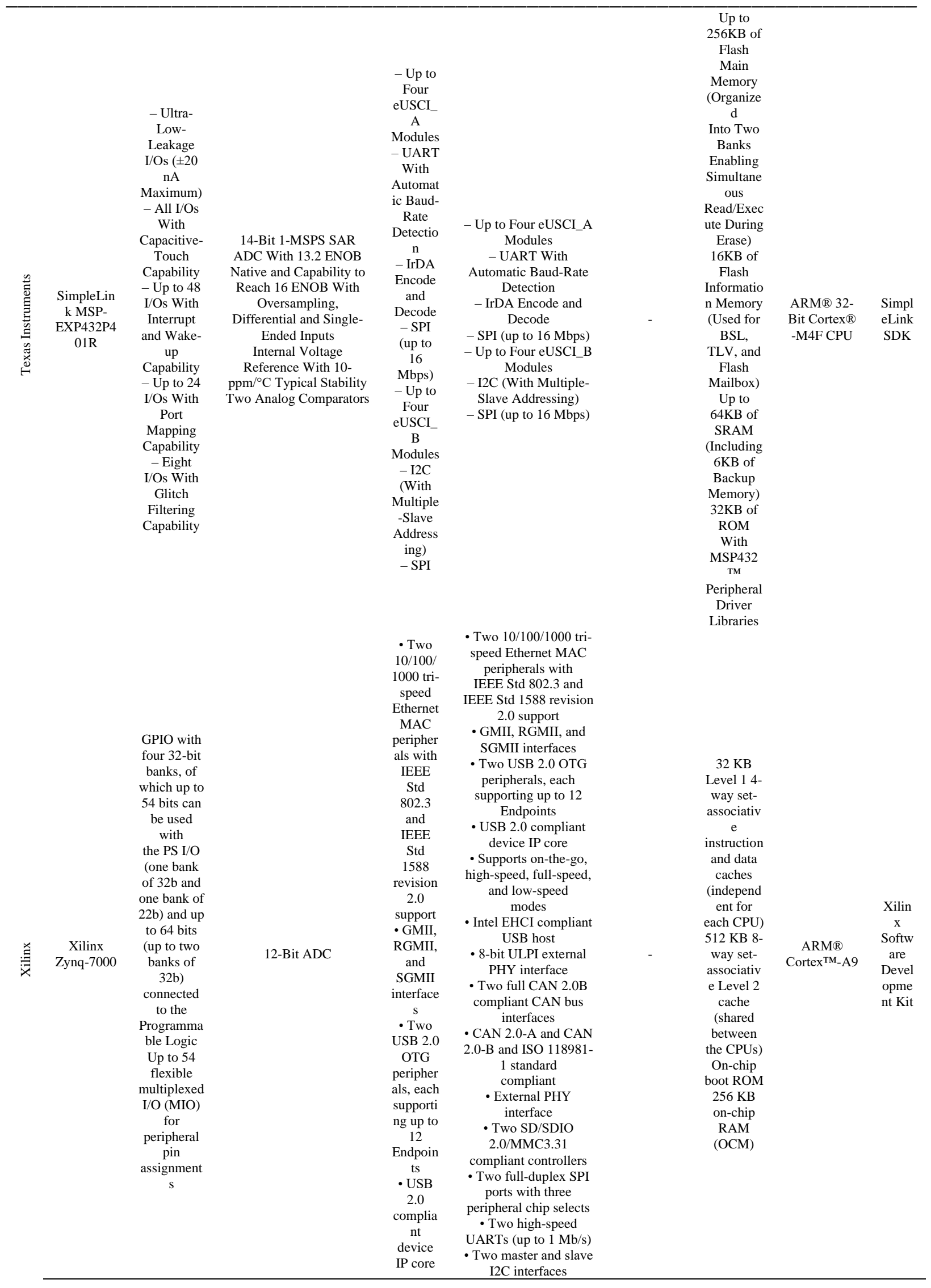

